\title{
The role of Jagged1/Notch pathway-mediated angiogenesis of hepatocarcinoma cells in vitro, and the effects of the spleen-invigorating and blood stasis-removing recipe
}

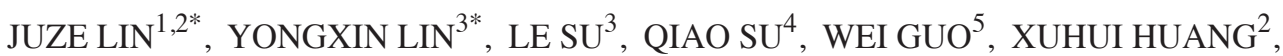 \\ CHANGJUN WANG ${ }^{2}$ and LIZHU LIN ${ }^{6}$
}

\begin{abstract}
${ }^{1}$ The First Clinical Medical College of Guangzhou University of Chinese Medicine;
${ }^{2}$ Department of Traditional Chinese Medicine, Guangdong General Hospital, Guangdong Academy of Medical Sciences, Guangdong Geriatric Institute; ${ }^{3}$ Guangzhou Traditional Chinese Medicine Hospital Affiliated to Guangzhou University of Chinese Medicine; ${ }^{4}$ Animal Experiment Center, The First Affiliated Hospital of Sun Yat-Sen University;

${ }^{5}$ Tropical Medicine Institute, Guangzhou University of Chinese Medicine; ${ }^{6}$ Cancer Center, The First Affiliated Hospital of Guangzhou University of Chinese Medicine, Guangzhou, Guangdong, P.R. China
\end{abstract}

Received May 31, 2017; Accepted July 5, 2017

DOI: 10.3892/ol.2017.6611

\begin{abstract}
The objective of this study was to observe the effect of Jagged1/Notch pathway-mediated angiogenesis on the in vitro proliferation of hepatocellular carcinoma cells, and the effect and possible mechanism of the spleen-invigorating and blood stasis-removing recipe. Spleen-invigorating and blood stasis-removing recipe serum from SPF grade nude mice was prepared, and the fingerprint of the drugs of the spleen-invigorating and blood stasis-removing recipe and drug serum were identified by HPLC. SMMC-7721 human hepatocellular carcinoma cells were divided into the normal control group, DAPT inhibitor control group, and drug serum group according to the different treatments. The Cell Counting Kit-8 (CCK-8) method was used to determine cell proliferation ability, and angiogenesis was observed under an inverted microscope. The expression of Jagged1, Notch1, and VEGF was measured by qPCR and western blot analysis. The interaction of Jagged1 and Notch1 was detected by Co-IP. The CCK-8 assay indicated that cell proliferation was inhibited in response to drug treatment $(\mathrm{P}<0.01)$. The expression of Jagged1, Notch1, and VEGF in the drug serum group was significantly lower than in the normal control group $(\mathrm{P}<0.01)$. Compared with the control group, the new vascular area of
\end{abstract}

Correspondence to: Dr Lizhu Lin, Cancer Center, The First Affiliated Hospital of Guangzhou University of Chinese Medicine, 12 Airport Road, Guangzhou, Guangdong 510405, P.R. China E-mail: 1v93pd@163.com

*Contributed equally

Key words: Jagged1, Notch, angiogenesis, hepatocellular carcinoma, spleen-invigorating and blood stasis-removing recipe, cell culture the DAPT inhibitor control group and drug serum group was smaller, and the blood vessels of the DAPT inhibitor control group and drug serum group were more sparse. The levels of Jagged1, Notch1, VEGF protein and the interaction between Jagged1 and Notch1 in the DAPT inhibitor control group and drug serum group were significantly lower than in the control serum group $(\mathrm{P}<0.01)$. In conclusion, the spleen-invigorating and blood stasis-removing recipe can inhibit the proliferation of hepatocellular carcinoma cells, and tumor angiogenesis in vitro. The function is related to the reduced expression of Jagged1, reduced interaction between Jagged1 and Notch1, and the reduced expression and activity of VEGF.

\section{Introduction}

The incidence of hepatocellular carcinoma in China is high, and surgical resection remains the most effective way to improve the long-term survival of patients. However, the recurrence rate of hepatocellular carcinoma after radical resection is as high as $50-70 \%$ or more, and the recurrence of liver cancer is the leading cause of death in patients (1). Postoperative recurrence is the greatest risk factor affecting prognosis. Hepatocellular carcinoma is a hypervascular malignant tumor. It was confirmed that the occurrence and development of liver cancer are closely related to angiogenesis. Inhibiting tumor angiogenesis can prevent the development of hepatocellular carcinoma $(2,3)$.

Notch is a group of receptor proteins expressed on the surface of vascular cells. Five Notch-matched ligands including Jagged1, Jagged2, Dll1, Dll3, and Dll4 have been identified in mammals. These five ligands can interact with Notch1, 2, 3, and 4 to activate the Notch pathway (4). The expression of Notch1 protein and mRNA is significantly higher in hepatocellular carcinoma tissue compared with adjacent normal liver tissue (5). A study of angiogenesis found that Notch can act as a 'switch' of vascular growth by interacting with different ligands (6). Recent studies found that Jagged1 is closely related 
to tumor formation, especially in regards to tumor angiogenesis. In the process of vessel sprouting, high expression of Jagged1 can increase the number of vascular endothelial cells, and enhance sprouting ability by inhibiting D114 to activate Notch (7). As an angiogenic factor, VEGF can stimulate endothelial cells to secrete a variety of factors, causing vascular basement membrane degradation, so that cells have sufficient space to migrate and proliferate, eventually leading to the formation of new blood vessels. Chinese medicine can be used to treat primary liver cancer. Previous clinical and experimental studies found that the spleen-invigorating and blood stasis-removing recipe can promote apoptosis of liver cancer cells (8-10). The aim of this study was to investigate the effect of the Jagged1-regulated Notch pathway on the proliferation and angiogenesis of hepatocellular carcinoma, and the effects of the spleen-invigorating and blood stasis-removing recipe, to provide a basis for its clinical application.

\section{Materials and methods}

Reagents. SMMC-7721 liver cancer cells (Shanghai Xinyu Biotechnology Co., Ltd., Shanghai, China); spleen-invigorating and blood stasis-removing recipe (12 g Curcuma, $20 \mathrm{~g}$ Atractylodes, 20 g Poria, 12 g Sophora, 12 g Bergamot, and $15 \mathrm{~g} \mathrm{Hedyotis} \mathrm{diffusa)} \mathrm{was} \mathrm{provided} \mathrm{by} \mathrm{the} \mathrm{Chinese} \mathrm{Pharmacy}$ of Guangdong Provincial People's Hospital (Guangzhou, China); fetal liver serum (Gibco, Shanghai, China); RPMI-1640 medium (Hyclone, Beijing, China); streptomycin, PBS buffer, and trypsin (Genview, Shanghai, China); Cell Counting Kit-8 (CCK-8) (Dojindo China Co., Ltd., Shanghai, China); Corning Matrigel Basement Membrane Matrix (Corning, Shanghai, China); DAPT inhibitor (AbMole BioScience, Shanghai, China); Tris-base, glycine, and sodium dodecyl sulfate (SDS) (Sangon Biotech Co., Ltd., Shanghai, China); acrylamide, BIS-acrylamide, PMSF, and Bromphenol Blue (Shanghai Genebase Gene-Tech Co., Ltd., Shanghai, China); BCA protein concentration measurement reagent (Thermo Fisher Scientific, Inc., Waltham, MA, USA); ammonium persulphate (Genview); TRIzol (Takara, Dalian, China); RT-PCR reagents and reverse transcription kit (DBI Bioscience, Shanghai, China); DEPC (Sigma-Aldrich, San Francisco, CA, USA); RNasin (Promega, Madison, WI, USA).

Equipment. Superclean bench (Suzhou Purification Equipment Co., Ltd., Suzhou, China); low speed centrifuge (Shenzhen Anke High-Tech Co., Ltd., Shenzhen, China); inverted microscope (Motic, Xiamen, China); $\mathrm{CO}_{2}$ cell incubator (Memmert Trading Co., Ltd., Shanghai, China); enzyme-linked immunosorbent detector (Thermo Fisher Scientific, Inc.); desktop high-speed centrifuge (Shanghai Anting Science Instrument Factory, Shanghai, China); constant pressure constant current electrophoresis instrument (Beijing Liuyi Instrument Factory, Beijing, China); frozen high-speed centrifuge (Zhuhai Heima Medical Instrument Co., Ltd., Zhuhai, China); magnetic stirrer (Changzhou Guohua Electric Appliance Co., Ltd., Changzhou, China); multi-purpose decolorization shaker (Jiangsu Xinkang Medical Devices Co., Ltd., Taizhou, China); ultrasonic crusher (Ningbo Xinzhi Biotechnology Co., Ltd., Ningbo, China); Swirl Oscillator (Shanghai Jingke Industrial Co., Ltd., Shanghai, China); SDS-PAGE vertical electrophoresis tank (Atobo,
Beijing, China); scanner (Shanghai Microtek Technology Co., Ltd., Shanghai, China); spectrophotometer (Shimadzu Co., Ltd., Beijing, China); gel scanning system (Zhongshan Golden Bridge, Beijing, China); air thermostat oscillator (Taicang Longway Medical Instrument Co., Ltd., Taicang, China); ABI 9700 PCR Amplifier (Applied Biosystems, Shanghai, China); Stratagene Mx3000P Real-time PCR instrument (Agilent Technologies, Palo Alto, CA, USA).

Preparation of drug serum. The composition of the spleen-invigorating and blood stasis-removing recipe (formerly known as 960 mixture) was as described above. The total weight of each prescription was $96 \mathrm{~g}$.

Preparation of the spleen-invigorating and blood stasis-removing recipe. The traditional Chinese medicines were collected according to the prescription ratio. A volatile oil extractor was used to extract volatile oil from curcuma, Atractylodes, and bergamot. Next, the residues of curcuma, Atractylodes, and bergamot were mixed with poria, Sophora flavescens, and Hedyotis diffusa, and were soaked in pure water (10 times volume) for $30 \mathrm{~min}$, and extracted three times at $90-100^{\circ} \mathrm{C}$, for $1.5 \mathrm{~h}$ each time. The extracts were combined, filtered, and concentrated, and finally mixed with the volatile oil. The ratio of drug to extract was calculated. The resulting extract was stored at $-20^{\circ} \mathrm{C}$ after disinfection by radioactive isotope, cobalt- 60 .

Determination of the dose. According to previous studies (11), the equivalent dose ratio calculated based on the body surface area (humans, $70 \mathrm{~kg}$; mice, $0.020 \mathrm{~kg}$ ) was 0.0026 . According to the conversion formula, the equivalent dose for mice was $12.48 \mathrm{~g} / \mathrm{kg}$ (96 g x 0.0026/0.020 kg = $12.48 \mathrm{~g} / \mathrm{kg}$ ). Therefore, we set the low, middle, and high dose of the spleen-invigorating and blood stasis-removing recipe as $6.24,12.48$, and $24.96 \mathrm{~g} / \mathrm{kg}$, respectively.

Administration method. The extract was harvested according to the ratio of drug to extract, and was mixed with saline to prepare an administration dose of $20 \mathrm{ml} / \mathrm{kg}$. Nude mice received intragastric administrations of saline containing the extract, and the administration volume was $20 \mathrm{ml} / \mathrm{kg}$. Mice were treated for 15 consecutive days. The mice in the normal control group were given equal volumes of saline.

Drug serum preparation. The same batch of SPF nude mice was randomly divided into the normal control group, spleen-invigorating and blood stasis-removing recipe high dose group, spleen-invigorating and blood stasis-removing recipe middle dose group, and spleen-invigorating and blood stasis-removing recipe low dose group, with 10 mice per group. The clinical dose was calculated according to a previous study (6). Saline or traditional Chinese medicine compounds were administered intragastrically for 15 days. Blood from the abdominal aorta was collected on day 16 , and serum was separated, filtered, and divided into smaller volumes for storage at $-80^{\circ} \mathrm{C}$.

Complete medium preparation. Based on our study, different concentrations of the spleen-invigorating and 
blood stasis-removing recipe had different inhibitory effects on the in vitro proliferation of hepatocarcinoma cells. The half-maximal inhibitory concentration $\left(\mathrm{IC}_{50}\right)$ and the inhibitory effects of the high dose of the spleen-invigorating and blood stasis-removing recipe $(24.96 \mathrm{~g} / \mathrm{kg})$ were both optimal among the four groups $(\mathrm{P}<0.05)$. Therefore, drug serum derived from the high dose treatment was used in this study.

Complete medium preparation for the normal serum group: RPMI-1640 medium $+10 \%$ normal nude mouse serum (from the normal control group) $+1 \%$ double antibiotics $(100 \mathrm{U} / \mathrm{ml}$ penicillin and $100 \mathrm{U} / \mathrm{ml}$ streptomycin). The preparation was performed at $4^{\circ} \mathrm{C}$, and the medium was used after being warmed in a $37^{\circ} \mathrm{C}$ water bath.

Complete medium preparation for the drug serum group: RPMI-1640 medium $+10 \%$ dosing nude mouse serum (from the spleen-invigorating and blood stasis-removing recipe high dose group $)+1 \%$ double antibiotics $(100 \mathrm{U} / \mathrm{ml}$ penicillin and $100 \mathrm{U} / \mathrm{ml}$ streptomycin). The preparation was performed at $4^{\circ} \mathrm{C}$, and the medium was used after being warmed in a $37^{\circ} \mathrm{C}$ water bath.

Complete medium preparation for the inhibitor group: RPMI-1640 medium $+10 \%$ normal nude mouse serum (from the normal control group) $+1 \%$ double antibiotics $(100 \mathrm{U} / \mathrm{ml}$ penicillin and $100 \mathrm{U} / \mathrm{ml}$ streptomycin) $+1 \mu \mathrm{M}$ of DAPT. The preparation was performed at $4^{\circ} \mathrm{C}$, and the medium was used after being warmed in a $37^{\circ} \mathrm{C}$ water bath.

HPLC analysis. Liquid phase conditions were used according to a previous study (12). The fingerprint of the drugs of the spleen-invigorating and blood stasis-removing recipe and drug serum were identified by HPLC at $323 \mathrm{~nm}$.

Cell resuscitation. The water bath was preheated to $37^{\circ} \mathrm{C}$; the frozen tube of cells was quickly placed in the heated water for rapid thawing; cells were transferred to a centrifuge tube, and RPMI-1640 complete medium was added, followed by centrifugation in a low-speed centrifuge. After centrifugation, cells were resuspended with medium, cell suspensions were added to cell culture flasks, and incubated in the incubator with $5 \% \mathrm{CO}_{2}$ at $37^{\circ} \mathrm{C}$.

Cell subculture. When approximately $80 \%$ of cells adhered to the wall of culture flasks, the culture medium was replaced under aseptic conditions. The culture conditions were $5 \% \mathrm{CO}_{2}$, saturated humidity, and $37^{\circ} \mathrm{C}$.

CCK-8-mediated detection of cell proliferation. The experimental grouping was the same as described above. The cultured SMMC-7721 cells were collected to prepare single cell suspensions under aseptic conditions, and the cell suspensions were adjusted to a density of $1 \times 10^{5} / \mathrm{ml}$. The cells were seeded in 96-well plates, with $100 \mu \mathrm{l}$ per well. Complete medium was added and the cells were cultured in an incubator $\left(37^{\circ} \mathrm{C}, 5 \% \mathrm{CO}_{2}\right)$ for $0,24,48$ and $72 \mathrm{~h}$. At the end of cell treatment, the culture solution was aspirated and $100 \mu \mathrm{l}$ of CCK-8 was added to each well without generation of air bubbles. The plates were incubated in the incubator for $4 \mathrm{~h}$. After, the medium was transferred to ELISA plates. The OD value at $450 \mathrm{~nm}$ was measured by an ELISA detector, and the data were analyzed.
Table I. qPCR reaction mixture.

\begin{tabular}{lr}
\hline Bestar $^{\circledR}$ SYBR-Green qPCR Master mix & $10 \mu 1$ \\
\hline PCR forward primer $(10 \mu \mathrm{M})$ & $0.5 \mu 1$ \\
PCR reverse primer $(10 \mu \mathrm{M})$ & $0.5 \mu 1$ \\
cDNA template & $1 \mu 1$ \\
ddH $_{2} \mathrm{O}$ & $8 \mu 1$ \\
Total & $20 \mu 1$ \\
\hline
\end{tabular}

Angiogenesis test. The experimental grouping was the same as described above. Matrigel stock solution was maintained at room temperature for melting overnight. Matrigel stock solution and serum-free RPMI-1640 medium were used to prepare a gel solution, and the solution was placed in 96-well plates, followed by incubation for $2 \mathrm{~h}$ for it to solidify. The cultured SMMC-7721 cells were collected and digested to prepare single cell suspensions under aseptic conditions, and the cell suspensions were adjusted to a density of $1 \times 10^{5} / \mathrm{ml}$. The cells were seeded in 96-well plates with $100 \mu \mathrm{l}$ per well. The plates were incubated in an incubator $\left(5 \% \mathrm{CO}_{2}, 37^{\circ} \mathrm{C}\right)$ for $6-8 \mathrm{~h}$, and a Motic inverted microscope was used to observe the cells and take pictures.

$q P C R$. RNA extraction: An appropriate amount of cells was collected and ground with liquid nitrogen. Total RNA extraction was performed using appropriate reagents. cDNA synthesis: A total of $1 \mu \mathrm{g}$ of total RNA was used as template, and the reaction system was prepared according to the instructions of the Bestar qPCR RT kit. Fluorescence quantification: The reaction was performed with a volume of $20 \mu \mathrm{l}$ (DBI Bestar $^{\circledR}$ SYBR-Green qPCR Master mix) using real-time PCR. The reaction system was prepared according to Table I. Reaction conditions for qPCR: $94^{\circ} \mathrm{C}$ for $2 \mathrm{~min}$, followed by 40 cycles of $94^{\circ} \mathrm{C}$ for $20 \mathrm{sec}, 58^{\circ} \mathrm{C}$ for $20 \mathrm{sec}$, and $72^{\circ} \mathrm{C}$ for $20 \mathrm{sec}$. Melting curve analysis: Temperature between $62^{\circ} \mathrm{C}$ and $95^{\circ} \mathrm{C}$. Fluorescence quantitative PCR was performed using Agilent Stratagene fluorescence quantitative PCR Mx3000P. The housekeeping gene, GAPDH, was used as the endogenous reference. Primer sequences are shown in Table II. The relative expression of target gene mRNA was calculated according to the $2^{-\Delta \Delta \mathrm{Cq}}$ method, where $\Delta \mathrm{Cq}=\mathrm{Cq}$ (target gene) $-\mathrm{Cq}$ (internal reference gene), $\Delta \Delta \mathrm{Cq}=\Delta \mathrm{Cq}$ (experimental group) $-\Delta \mathrm{Cq}$ (control group).

Western blot analysis. Cells were lysed with RIPA buffer, and the concentration of the protein lysate was determined using a BCA protein quantification kit. Total protein $(20 \mu \mathrm{g})$ from each group was electrophoresed using $10 \%$ SDS-PAGE for $2 \mathrm{~h}$ at $120 \mathrm{~V}$ constant voltage. Total protein was transferred to NC membranes. Membranes were then incubated with rabbit polyclonal Notch1 antibody (dilution, 1:1,000; cat. no. ab27526); rabbit monoclonal VEGF antibody (dilution, 1:1,000; cat. no. ab109536) and rabbit monoclonal Jagged1 antibody (dilution, 1:1,000; cat. no. ab32152), which were all purchased from Abcam (Cambridge, MA, USA) at $4^{\circ} \mathrm{C}$ overnight. The membranes were washed three times, and the corresponding secondary antibody was added and incubated for $2 \mathrm{~h}$ followed 
Table II. Primer sequences.

\begin{tabular}{llc}
\hline ID & \multicolumn{1}{c}{ Sequence $\left(5^{\prime} \rightarrow 3^{\prime}\right)$} & $\begin{array}{c}\text { Product } \\
\text { length }(\mathrm{bp})\end{array}$ \\
\hline Jagged1 & F: GCCTGGCCGAGGTCCTATA & 144 \\
& R: CGTGTTCTGCTTCAGCGTCT & \\
Notch1 & F: CCGCCTTTGTGCTTCTGTT & 127 \\
& R: CGCCGCTTCTTCTTGCTG & \\
VEGF & F: GCCTTGCCTTGCTGCTCTAC & 146 \\
& R: CACCAGGGTCTCGATTGGAT & \\
GAPDH & F: ACACCCACTCCTCCACCTTT & 154 \\
& R: TTACTCCTTGGAGGCCATGT & \\
& &
\end{tabular}

by three washes. ECL luminescence solution was added for chemiluminescence, exposure, and development. X-film was used for exposure and scanned, and the net density value was analyzed using a gel image processing system (Image-Pro Plus 6.0; QMgene, Beijing, China).

Co-IP to detect the interaction between Jaggedl and Notch1. Cells were collected, protein was extracted, and the concentration was measured using the Co-IP kit. A total of $100 \mu \mathrm{l}(300 \mu \mathrm{g})$ of the protein extract and beads that were previously combined with the Notch 1 antibody were mixed and incubated at $4^{\circ} \mathrm{C}$ overnight. The obtained pure protein and antibody complex was washed and eluted for western blot detection.

Statistical analysis. Data were analyzed using SPSS13.0 statistical software (SPSS, Inc., Chicago, IL, USA). Numerical data are presented as false $\pm \mathrm{SD}$. Comparisons between two groups were by two-sample t-test. Comparisons within a group were by paired sample t-test.

\section{Results}

HPLC analysis. HPLC analysis showed that drug serum contained the ingredients of the spleen-invigorating and blood stasis-removing recipe (Fig. 1).

CCK-8-mediated detection of cell proliferation. SMMC-7721 cells were cultured in serum, drug serum, and serum containing inhibitor, and cell proliferation was detected using the CCK-8 method at $0,24,48$ and $72 \mathrm{~h}$, respectively. There was no significant difference in OD value between the three groups at $0 \mathrm{~h}$ ( $P>0.05)$, although significant differences were found at 24, 48, and $72 \mathrm{~h}(\mathrm{P}<0.01)$ (Fig. 2).

Angiogenesis test. The normal serum group, drug serum group, and inhibitor group were observed under an inverted microscope (x200). Compared with the drug serum group and inhibitor group, SMMC-7721 cells in the normal serum group formed a larger area of new blood vessels with denser blood vessels (Fig. 3).

Measurement of Jagged1, Notch1, and VEGF mRNA by qPCR. qPCR analysis showed that the levels of Jagged1 (Fig. 4A), Notch1 (Fig. 4B), and VEGF (Fig. 4C) in SMMC-7721 cells
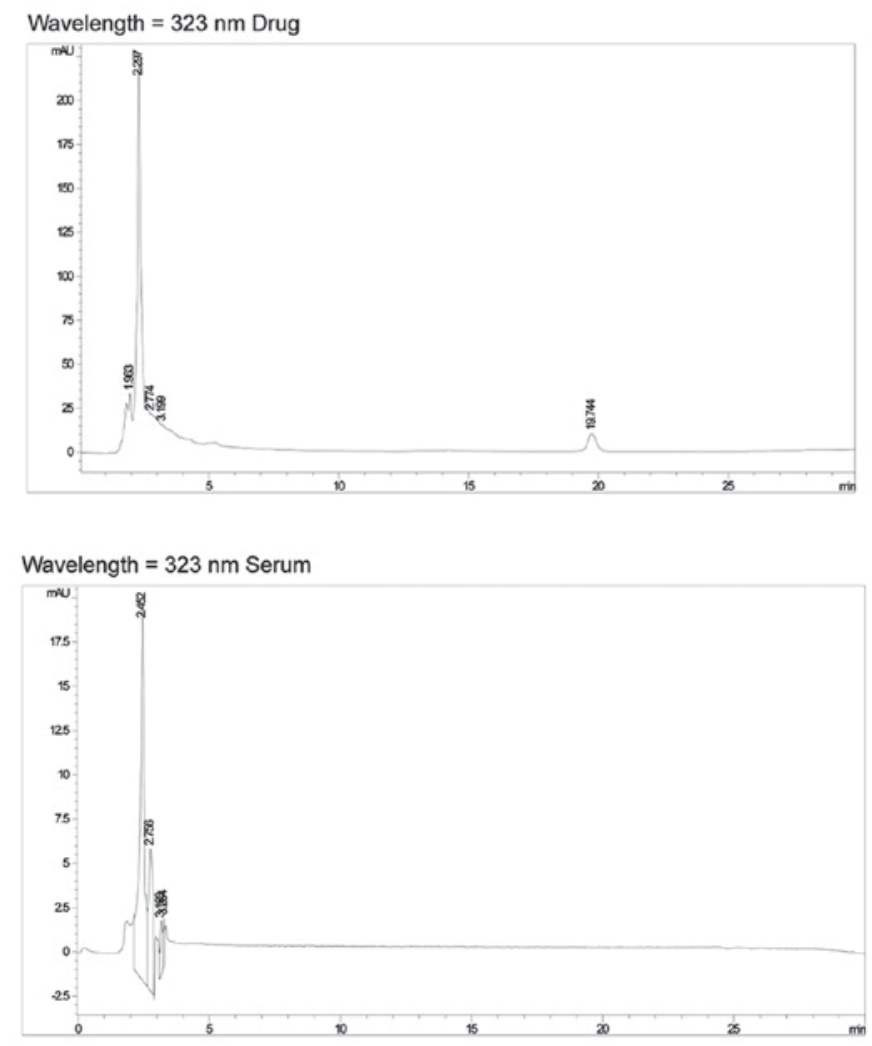

Figure 1. Fingerprint of the drugs of the spleen-invigorating and blood stasis-removing recipe and drug serum. HPLC analysis showed that drugs of the spleen-invigorating and blood stasis-removing recipe and drug serum showed similar peaks within 0-5 min.

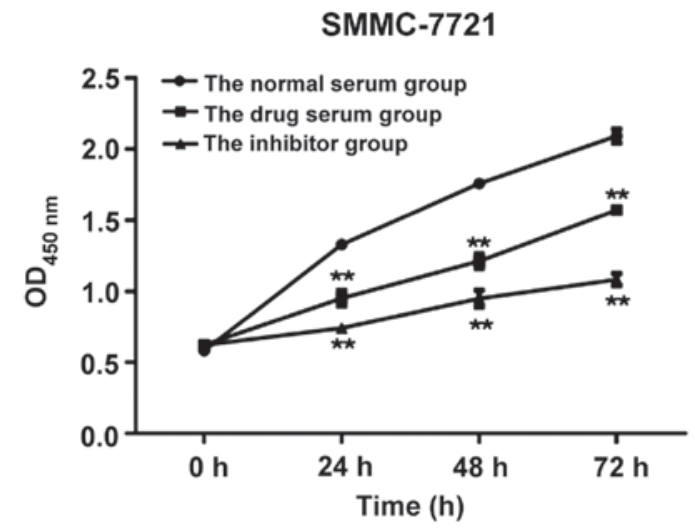

Figure 2. The proliferation of the three groups of SMMC-7721 cells at the four time points. Compared with the normal serum control group, ${ }^{* *} \mathrm{P}<0.01$.

of the drug serum group and inhibitor group were significantly lower than those of the normal serum group $(\mathrm{P}<0.01)$.

The expression of Jagged1, Notch1, and VEGF protein by western blot analysis. The expression of Jagged1, Notch1, and VEGF protein in SMMC-7721 cells was measured by western blot analysis. The levels of Jagged1, Notch1, and VEGF protein in SMMC-7721 cells of the drug serum group were significantly lower than those of the normal serum group $(\mathrm{P}<0.01)$. The levels of Jagged1 and Notch1 were decreased after adding the Notch inhibitor, DAPT, indicating that DAPT successfully inhibited the Jagged1/Notch signaling pathway. 


\section{The normal serum group}

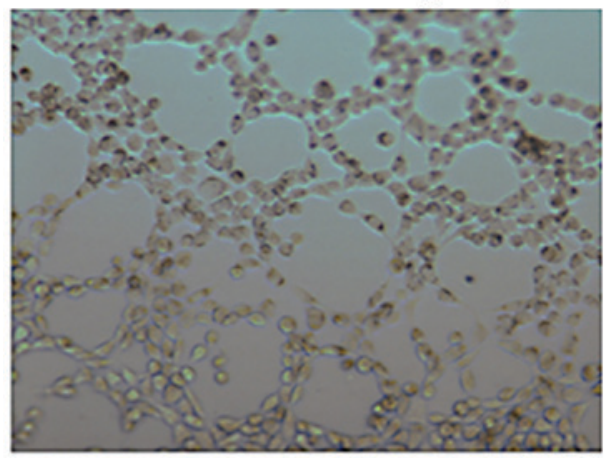

The inhibitor group

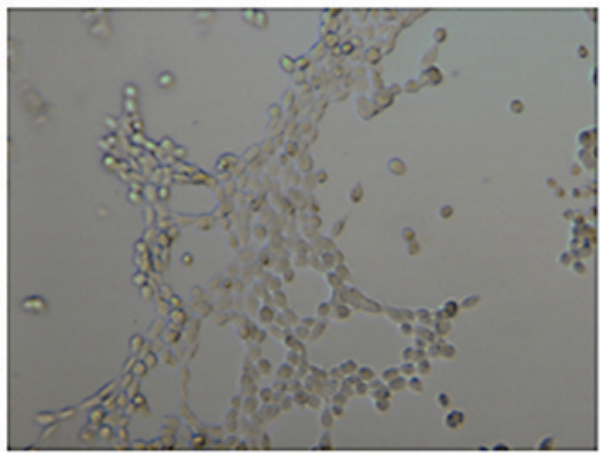

The drug serum group

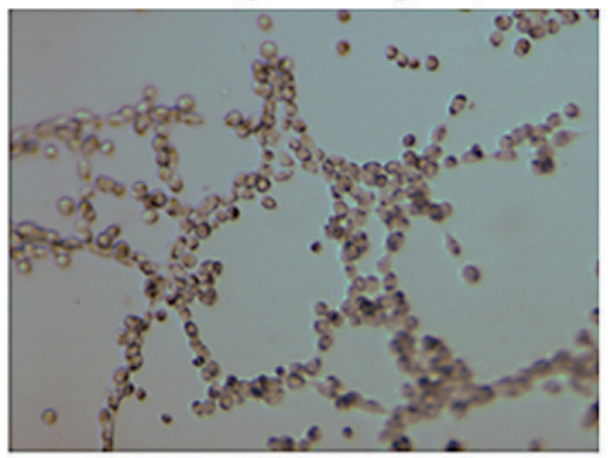

Angiogenesis

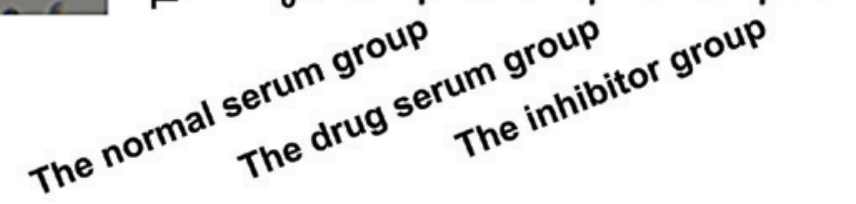

Figure 3. Comparison of angiogenesis between the normal serum group, drug serum group, and inhibitor serum group. Angiogenesis test showed that compared with the normal serum group, the drug serum group and inhibitor group had fewer and sparser new blood vessels compared with the normal serum group. ${ }^{* *} \mathrm{P}<0.01$.

A

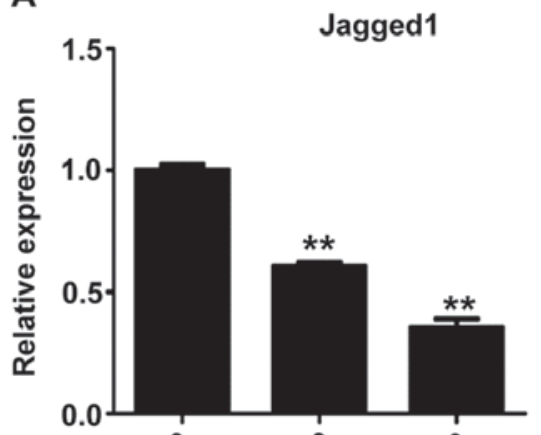

B

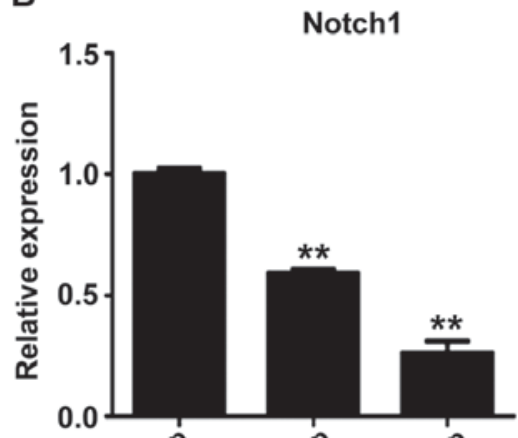

C

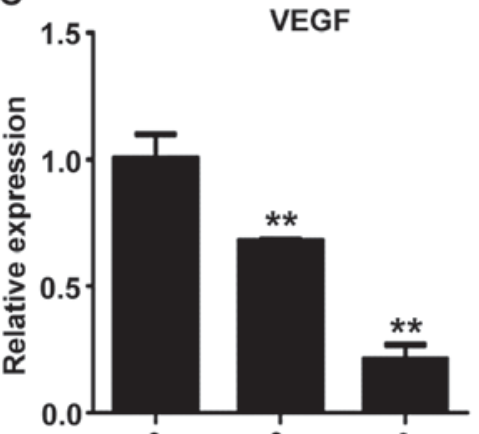

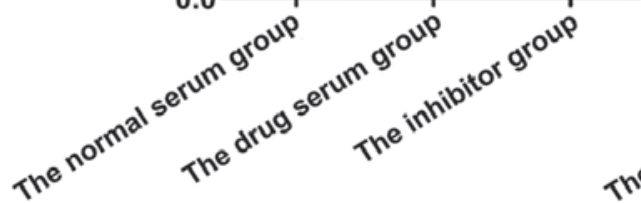
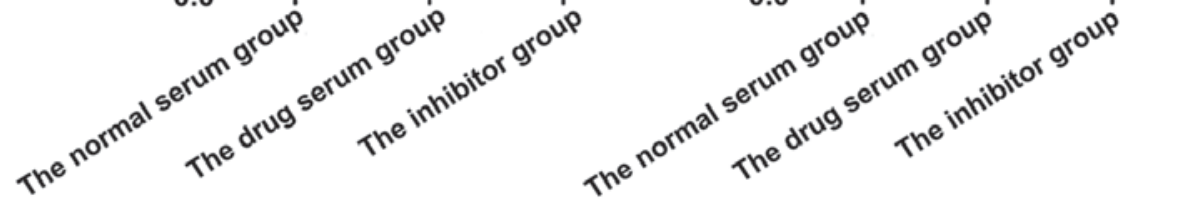

Figure 4. Comparison of (A) Jagged1, (B) Notch1, and (C) VEGF expression between the normal serum group, drug serum group, and inhibitor group. Compared with the normal serum group, ${ }^{* *} \mathrm{P}<0.01$. The expression of Jagged1, Notch1, and VEGF in SMMC-7721 hepatocellular carcinoma cells was inhibited in the inhibitor group, and the inhibitory effect of the drug serum was similar to that of the inhibitor.

In addition, the expression of VEGF protein was reduced after adding DAPT (Fig. 5).

The interaction between Jaggedl and Notchl detected by Co-IP. The interaction between Jagged 1 and Notch 1 in
SMMC-7721 cells of the drug serum group was significantly lower than that in the normal serum group $(\mathrm{P}<0.01)$. The interaction between Jagged1 and Notch1 in SMMC-7721 cells was also significantly reduced after adding DAPT compared with that of the normal serum group $(\mathrm{P}<0.01)$ (Fig. 6). 

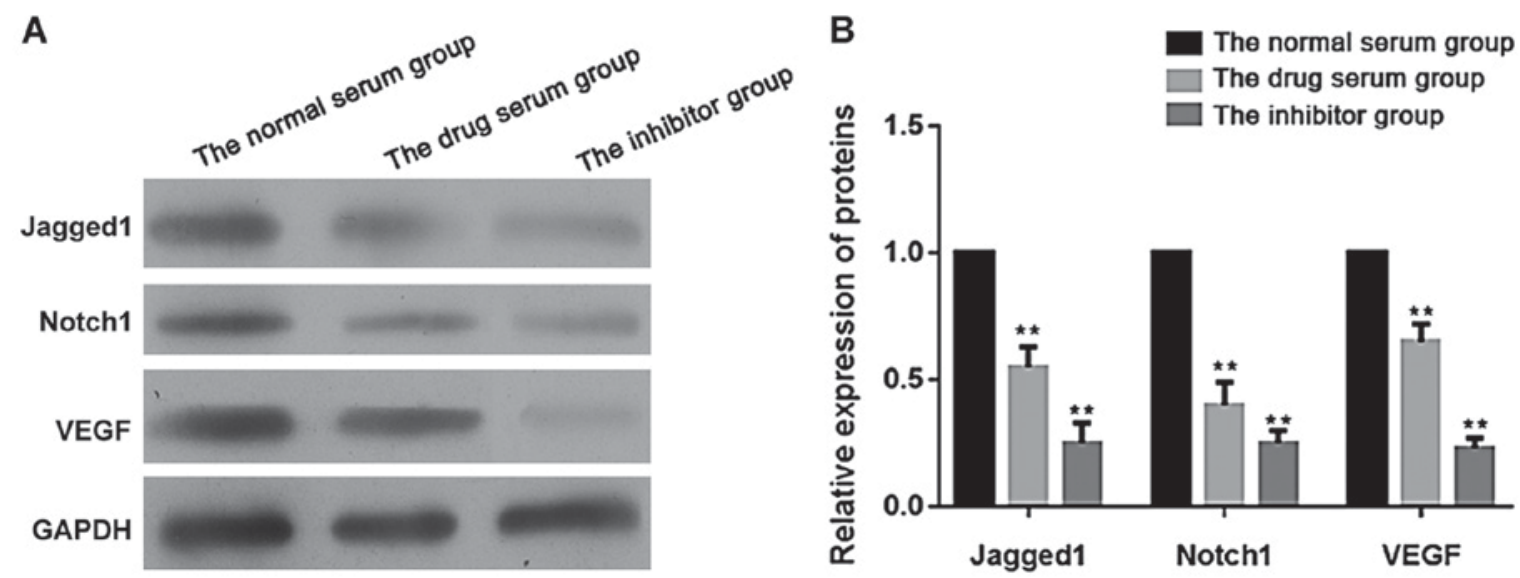

Figure 5. Comparison of Jagged1, Notch1, and VEGF expression between the normal serum group, drug serum group, and inhibitor group. (A and B) Western blot analysis shows that the levels of Jagged1, Notch1, and VEGF in SMMC-7721 cells of the drug serum group and inhibitor group were significantly lower than those in SMMC-7721 cells of the normal serum control group. ${ }^{* *} \mathrm{P}<0.01$.

A

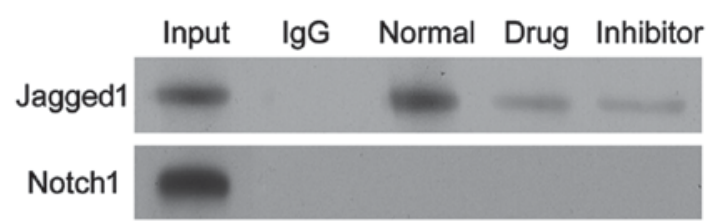

B

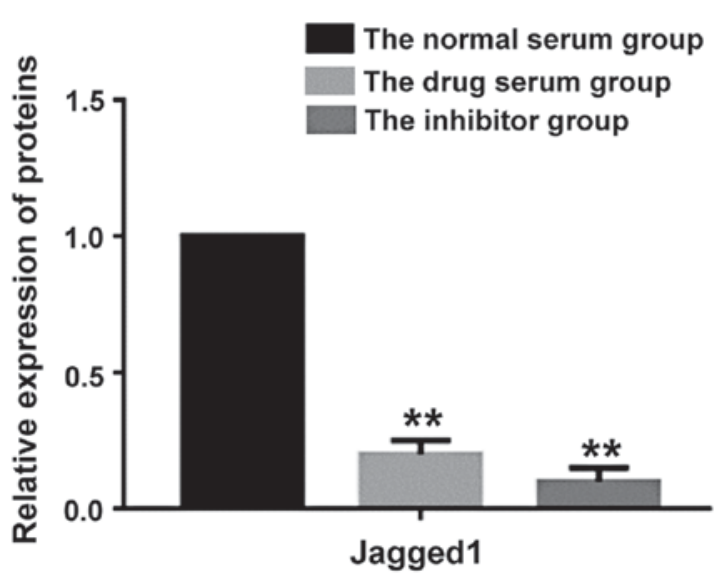

Figure 6. Comparison of the interaction between Jagged1 and Notch1 in the normal serum group, drug serum group, and inhibitor group. (A) Western blotting and (B) Co-IP assay shows that the interaction between Jagged1 and Notch1 in SMMC-7721 cells of the drug serum group and inhibitor group were both significantly lower than in SMMC-7721 cells of the normal serum control group. ${ }^{* *} \mathrm{P}<0.01$.

\section{Discussion}

Hepatocellular carcinoma is associated with one of the highest rates of mortality among all diseases worldwide. The number of new cases of liver cancer and the number of deaths in China account for about half of the global total. In 1971, Folkman (13) first reported that tumor growth and metastasis are dependent on angiogenesis. The regulation of tumor angiogenesis and anti-angiogenesis in the treatment of cancer has become an area of intense research. It was found that the positive expression rates of Notch1 and Jagged1 in human hepatocellular carcinoma are higher than those in adjacent cirrhotic tissue and normal tissue $(\mathrm{P}<0.05)$. The expression of Notch1 in hepatocellular carcinoma is closely related to the expression of Jagged1 $(\mathrm{P}<0.05)$, suggesting that Notch1 and Jagged1 can promote the development of hepatocellular carcinoma (14). The Notch signaling pathway has been shown to play an important role in the development and differentiation of endothelial cells, as well as in angiogenesis, and tumor development $(15,16)$. As an important ligand in the Notch signaling pathway, Jagged1 can bind activated Notch1 to antagonize D114 activity, enhance VEGF activity, and promote its expression. This in turn stimulates tumor neovascularization and promotes tumor growth, invasion, and metastasis (6). The discovery of this phenomenon promoted a new approach to study the relationship between the growth of hepatocellular carcinoma and angiogenesis, and provides new insights into the comprehensive treatment of hepatocellular carcinoma.

The spleen-invigorating and blood stasis-removing recipe (formerly known as 960 mixture or the Fuzheng Kangai recipe, contains Atractylodes Rhizome, curcuma, poria, bergamot, Sophora flavescens, and Hedyotis diffusa, with a total of $96 \mathrm{~g}$ ) is used by our department for treatment after liver cancer surgery. This recipe is derived from the prescription of Professor Qian Bowen, a famous traditional Chinese medical doctor at Shanghai University of Traditional Chinese Medicine. With heat clearing-detoxication ability, this recipe can replenish qi to invigorate the spleen. An increasing number of studies found that many traditional Chinese medicines or their compounds have antitumor effects $(17,18)$. Some scholars further determined that many traditional Chinese medicines can regulate the immune system to inhibit tumor neovascularization, which in turn inhibits metastasis (19).

HPLC was used to analyze the fingerprint of the drugs of the spleen-invigorating and blood stasis-removing recipe and drug serum at $323 \mathrm{~nm}$. HPLC analysis showed that drugs of the spleen-invigorating and blood stasis-removing recipe and drug serum showed similar peaks within 0-5 min, indicating that drug serum contained the ingredients of the spleen-invigorating and blood stasis-removing recipe. SMMC-7721 hepatocarcinoma cells were cultured in vitro in medium containing normal serum or drug serum. The proliferation of SMMC-7721 cells was detected at $0,24,48$ and $72 \mathrm{~h}$, respec- 
tively. The proliferation of hepatocellular carcinoma cells was significantly inhibited in the drug serum group $(\mathrm{P}<0.01)$ in a time-dependent manner compared with the normal serum group, indicating that the spleen-invigorating and blood stasis-removing recipe can inhibit the in vitro proliferation of SMMC-7721 cells. Angiogenesis of hepatocellular carcinoma cells in vitro was observed under an inverted microscope. Angiogenesis test showed that compared with the normal serum group, the drug serum group and inhibitor group had fewer and more sparse new blood vessels. Similar results were found in the drug serum group and inhibitor serum group, indicating that the spleen-invigorating and blood stasis-removing recipe can inhibit the angiogenesis of liver cancer cells.

DAPT inhibits the activity of the Notch1 pathway mainly by inhibiting $\gamma$-secretase (20). In this study, we further analyzed the possible mechanism of the spleen-invigorating and blood stasis-removing recipe in inhibiting the angiogenesis of hepatocarcinoma cells in vitro. In our study, SMMC-7721 cells were cultured in vitro, and the levels of Jagged1, Notch1, and VEGF in the normal serum group, drug serum group, and inhibitor serum control group were measured by qPCR and western blot analysis. In addition, the interaction between Jagged1 and Notch1 was detected by Co-IP. The levels of Jagged1, Notch1, and VEGF mRNA and protein in SMMC-7721 cells were significantly lower in the drug serum group and inhibitor serum group compared with the normal serum group $(\mathrm{P}<0.01)$. The interaction of Jagged1 and Notch1 in SMMC-7721 cells was significantly lower in the drug serum group than the normal serum group $(\mathrm{P}<0.01)$. Similar results were observed in the inhibitor serum group with the addition of the Notch signaling pathway inhibitor (DAPT).

In conclusion, we confirmed that the spleen-invigorating and blood stasis-removing recipe can significantly inhibit the proliferation and angiogenesis of hepatocellular carcinoma cells in vitro. The function of the spleen-invigorating and blood stasis-removing recipe is related to the reduced expression of Jagged1, reduced interaction between Jagged1 and Notch1, and the reduced expression and activity of VEGF, to inhibit tumor neovascularization, and ultimately inhibit the growth, invasion, and metastasis of liver cancer. To further validate our results, we will develop a postoperative dormant animal model of hepatocellular carcinoma to study the effects of Jagged1/Notch1 pathway-mediated angiogenesis on liver cancer dormancy, and the spleen-invigorating and blood stasis-removing recipe.

\section{Acknowledgements}

The present study was supported by the Fund of Building Strong TCM Province of Traditional Chinese Medicine Bureau of Guangdong Province (project no. 20141001).

\section{References}

1. Catto JW, Alcaraz A, Bjartell AS, De Vere White R, Evans CP, Fussel S, Hamdy FC, Kallioniemi O, Mengual L, Schlomm T and Visakorpi T: MicroRNA in prostate, bladder, and kidney cancer: A systematic review. Eur Urol 59: 671-681, 2011.

2. Fabregat I, Roncero C and Fernández M: Survival and apoptosis: A dysregulated balance in liver cancer. Liver Int 27: 155-162, 2007.
3. Yan SL, Huang CY, Wu ST and Yin MC: Oleanolic acid and ursolic acid induce apoptosis in four human liver cancer cell lines. Toxicol In Vitro 24: 842-848, 2010.

4. Hainaud P, Contrerès JO, Villemain A, Liu LX, Plouët J, Tobelem G and Dupuy E: The role of the vascular endothelial growth factor-Delta-like 4 ligand/Notch4-ephrin B2 cascade in tumor vessel remodeling and endothelial cell functions. Cancer Res 66: 8501-8510, 2006 .

5. Qi R, An H, Yu Y, Zhang M, Liu S, Xu H, Guo Z, Cheng T and Cao X: Notch1 signaling inhibits growth of human hepatocellular carcinoma through induction of cell cycle arrest and apoptosis. Cancer Res 63: 8323-8329, 2003.

6. Benedito R, Roca C, Sörensen I, Adams S, Gossler A, Fruttiger M and Adams RH: The notch ligands D1l4 and Jagged1 have opposing effects on angiogenesis. Cell 137: 1124-1135, 2009.

7. Kume T: Novel insights into the differential functions of Notch ligands in vascular formation. J Angiogenes Res 1: 8, 2009.

8. Di Carlo JV and Alexander SR: Hemofiltration for cytokine-driven illnesses: The mediator delivery hypothesis. Int J Artif Organs 28: 777-786, 2005.

9. Li S, Lin H, Tang Y, Li W, Shen J, Kai J, Yue S, Shang G, Zhu Z, Shang E, et al: Comparative metabolomics analysis on invigorating blood circulation for herb pair Gui-Hong by ultra-high-performance liquid chromatography coupled to quadrupole time-of-flight mass spectrometry and pattern recognition approach. J Pharm Biomed Anal 107: 456-463, 2015.

10. Tian S, Tang J,Liu H, Wang L, Shen J,Li J and Gan Y: Propyl gallate plays a nephroprotective role in early stage of diabetic nephropathy associated with suppression of glomerular endothelial cell proliferation and angiogenesis. Exp Diabetes Res 2012: 209567, 2012.

11. Sherry N,Hagopian W, Ludvigsson J, Jain SM, Wahlen J,Ferry RJ Jr, Bode B, Aronoff S, Holland C, Carlin D, et al; Protégé Trial Investigators: Teplizumab for treatment of type 1 diabetes (Protégé study): 1-year results from a randomised, placebo-controlled trial. Lancet 378: 487-497, 2011.

12. Jiang HL, Singh SK, Yan JM, Zhang XB and Xu Q: Liquid-phase chemical hydrogen storage: Catalytic hydrogen generation under ambient conditions. ChemSusChem 3: 541-549, 2010.

13. Folkman J: Tumor angiogenesis: Therapeutic implications. N Engl J Med 285: 1182-1186, 1971.

14. Wang M,Xue L, Cao Q,Lin Y,Ding Y, Yang P and Che L: Expression of Notch1, Jagged1 and beta-catenin and their clinicopathological significance in hepatocellular carcinoma. Neoplasma 56: 533-541, 2009.

15. Bray SJ: Notch signalling: A simple pathway becomes complex. Nat Rev Mol Cell Biol 7: 678-689, 2006.

16. Poon RT, Fan ST and Wong J: Clinical significance of angiogenesis in gastrointestinal cancers: A target for novel prognostic and therapeutic approaches. Ann Surg 238: 9-28, 2003.

17. Yang X and Wu XZ: Main anti-tumor angiogenesis agents isolated from Chinese herbal medicines. Mini Rev Med Chem 15: 1011-1023, 2015.

18. Tu X, Deng Y, Chen J, Hu Q, He C, Jordan JB and Zhong S: Screening study on the anti-angiogenic effects of Traditional Chinese Medicine - Part I: Heat-clearing and detoxicating TCM. J Ethnopharmacol 194: 280-287, 2016.

19. Fan G, Zong W and Zuo J: Dynamic observation and clinical significance of integrated traditional Chinese and Western medicine on interleukin-2 system, T cell and erythrocyte immune system in patients of lung cancer. Zhongguo Zhong Xi Yi Jie He Za Zhi 20: 586-588, 2000 (In Chinese).

20. Meng RD, Shelton CC, Li YM, Qin LX, Notterman D, Paty PB and Schwartz GK: gamma-Secretase inhibitors abrogate oxaliplatin-induced activation of the Notch-1 signaling pathway in colon cancer cells resulting in enhanced chemosensitivity. Cancer Res 69: 573-582, 2009. 